\title{
Long-term outcomes of the Bankart and Latarjet repairs: a systematic review
}

This article was published in the following Dove Press journal:

Open Access Journal of Sports Medicine

15 April 2017

Number of times this article has been viewed

\section{Natalie C Rollick' \\ Yohei Onol,2 \\ Hafeez M Kurji ${ }^{3}$ \\ Atiba A Nelson' \\ Richard S Boorman' \\ Gail M Thornton ${ }^{1,4}$ \\ Ian KY Lo'}

'Department of Surgery, Section of Orthopaedic Surgery, McCaig Institute for Bone and Joint Health, University of Calgary, Calgary, AB, Canada;

${ }^{2}$ Department of Orthopedic Surgery, Nagoya University Graduate School of Medicine, Nagoya, Japan; ${ }^{3}$ College of Medicine, University of Saskatchewan, Saskatoon, SK, ${ }^{4}$ Department of Orthopaedics, University of British Columbia, Vancouver, BC, Canada
Correspondence: lan KY Lo Department of Surgery, University of Calgary, 3330 Hospital Drive, NW,

Calgary, AB T2N IN4, Canada

Tel +l 4032202745

Fax +l 4032108197

Email ikylo@ucalgary.ca
Abstract: The most common surgical techniques for the treatment of recurrent anterior shoulder instability include the arthroscopic Bankart repair, the open Bankart repair and the open Latarjet procedure. The purpose of this study was to evaluate and compare the longterm outcomes following these procedures. A systematic review of modern procedures with a minimum follow-up of 5 years was completed. The objective outcome measures evaluated were post-operative dislocation and instability rate, the Rowe score, radiographic arthritis and complications. Twenty-eight studies with a total of 1652 repairs were analyzed. The estimated re-dislocation rate was $15.1 \%$ following arthroscopic Bankart repair, $7.7 \%$ following open Bankart repair and $2.7 \%$ following Latarjet repair, with the comparison between arthroscopic Bankart and open Latarjet reaching statistical significance $(p<0.001)$. The rates of subjective instability and radiographic arthritis were consistently high across groups, with no statistical difference between groups. Estimated complication rates were statistically higher in the open Latarjet repair $(9.4 \%)$ than in the arthroscopic Bankart $(0 \% ; p=0.002)$. The open Latarjet procedure yields the most reliable method of stabilization but the highest complication rate. There are uniformly high rates of post-operative subjective instability symptoms and radiographic arthritis at 5 years regardless of procedure choice.

Keywords: Bankart, Latarjet, long-term outcomes, instability

\section{Introduction}

The shoulder joint has the largest range of motion of all joints with little inherent bony stability. As such, it is one of the most frequently dislocated joints in the body with an incidence of up to 47 per 100,000 person years. ${ }^{1}$ Anterior glenohumeral dislocation commonly results in a number of pathologic lesions, including traumatic detachment of the anteroinferior capsulolabral complex from the glenoid rim (Bankart lesion ${ }^{2}$ ), stretching of the anterior capsule and ligamentous structures and damage to the bony glenoid or humeral head. Non-operative management has been associated with acceptable results, but recurrent dislocation can be as high as $90 \%$ in some patient populations. ${ }^{3}$ Multiple surgical procedures have been described to improve shoulder stability, each with a large number of technical variations, which in part has paralleled the advancement of surgical technology.

The Bankart repair ${ }^{2}$ focuses on the anatomical re-attachment of the labrum to the glenoid rim and has been described initially utilizing an open technique and more recently incorporating arthroscopic techniques as well. While initial rates of recurrent instability were high with arthroscopic trans-glenoid suture or tack repairs, success has 
dramatically improved with the addition of modern suture anchors. ${ }^{4}$ However, rates of recurrent instability remain as high as $10-22.5 \%{ }^{5,6}$ in some case series. Recurrent instability is particularly prevalent in the young patients as well as patients with significant bone loss. ${ }^{7,8}$

The Latarjet procedure can improve anterior stability by multiple mechanisms. Not only can the Bankart lesion be repaired and provide stability, but the transfer of the coracoid process extends the bony articular arc of the glenoid, and the addition of the conjoint tendon may provide dynamic stability as well. ${ }^{9}$ This procedure addresses the issue of glenoid bone loss that has recently been identified as a key factor in recurrent instability ${ }^{10,11}$ and has been shown to be superior to Bankart repairs in biomechanical studies. ${ }^{12}$ Multiple authors have described excellent clinical outcomes and stability rates following Latarjet reconstruction. However, the Latarjet results in significant distortion in normal anatomy and may be associated with a significant restriction in postoperative range of motion, ${ }^{13-15}$ a result that has been linked with high rates of osteoarthritis. ${ }^{16,17}$ Though focus is often directed toward coracoid transfer procedures, recent studies have shown that Bankart-type procedures may also result in restricted range of motion and a potentially increased risk for arthritis as well. ${ }^{18}$

Although numerous publications exist on the success of each technique, there are very few comparative trials evaluating these techniques, and very little evidence exists to examine the long-term outcomes of either procedure. Therefore, the purpose of this study was to compare the longterm clinical and radiographic outcomes of the arthroscopic Bankart, open Bankart and Latarjet repairs.

\section{Methods}

This review of literature was performed using the Preferred Reporting Items for Systemic Reviews and Meta-Analyses (PRISMA) guidelines and checklist (Figure 1). Two independent investigators conducted the search strategy involving PubMed, MEDLINE, Embase and Cochrane databases. Search terms included ("shoulder" OR "glenohumeral joint") AND (“dislocation" OR “subluxation" OR "instability") AND ("treatment" OR "surgery"). Inclusion criteria were 1) anterior glenohumeral instability, 2) Englishlanguage studies, 3) Bankart procedures using anchor-based or trans-osseous fixation (whether arthroscopic or open) and/or Latarjet procedures and 4) studies with a minimum of 5-year follow-up. Bankart procedures were defined as re-attachment of the anteroinferior labrum and capsule to the bony glenoid through anchors or trans-osseous fixation. Latarjet procedures were defined as those involving bony reconstruction of the anterior glenoid through transfer of the coracoid. Studies were excluded if they involved 1) Caspari's trans-glenoid fixation or tack-based fixation, 2) acute glenoid fractures, 3) posterior or multi-direction instability and 4) revision procedures. Studies were evaluated using the levels of evidence as outlined by the Oxford Centre for Evidence-Based Medicine. Two independent investigators conducted the title, abstract and article review independently. In the event there was disagreement regarding the inclusion of a study, a third investigator, the senior author, made the final decision. The final literature search was conducted on June 30, 2015. All references were cross-referenced to avoid omitting relevant studies. For studies using duplicate patient populations, only the most recent publication was used for analysis. The Coleman methodology score (CMS) ${ }^{19}$ was used to assess the quality of the studies.

The primary outcome is recurrent dislocation or instability symptoms. Instability symptoms included a broad clinical presentation, including subluxations, persistent apprehension, subjective sense of instability and recurrent dislocation events. Secondary outcomes include Rowe score, ${ }^{20}$ radiographic osteoarthritis and complication rate. Radiographic osteoarthritis was defined using the Samilson-Prieto classification, ${ }^{21}$ as this was the most commonly reported classification system. Grade one was considered mild, grade two as moderate and grade three as severe. One study did not formally employ the Samilson-Prieto classification but used similar gradations with mild, moderate and severe distinctions. For the purpose of this study, complications included general surgical complications (i.e., infection, neurologic injury) and procedure-specific complications (i.e., graft osteolysis, anchor-related complications). However, recurrent instability was not included as a complication as this was separately analyzed.

Analysis was a random-effects model using binomial within-study variance for all the outcome measures, except for the Rowe score, where a fixed-effects model weighted by sample size was applied. The group comparison outcomes were estimated via least squares mean values and compared between groups with a Bonferroni adjustment for multiple comparisons. Estimated mean scores or rates are listed under least squares mean values, while differences between groups on those are listed under least squares mean values for effect group. 


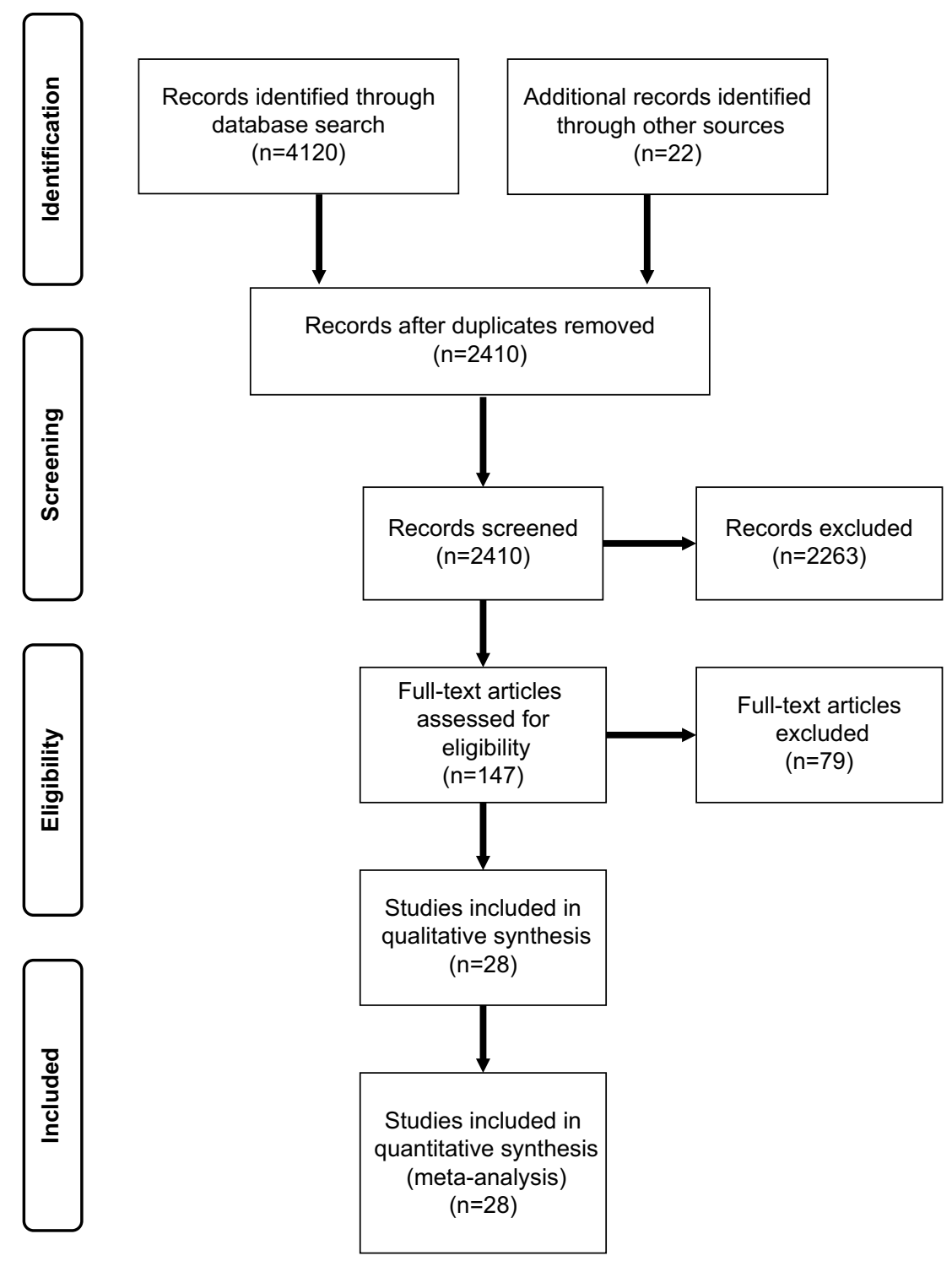

Figure I Systemic review algorithm using PRISMA guidelines.

Abbreviation: PRISMA, Preferred Reporting Items for Systemic Reviews and Meta-Analyses.

\section{Results}

A total of 4142 articles were identified by the literature search and reference review, and 2416 remained after removal of duplicates. Title review was completed to exclude foreign language papers, animal or biomechanical data, imaging studies and papers on unrelated topics. A total of 147 articles remained for abstract review and assessment of inclusion criteria (Figure 1). The most common reason for exclusion at this level was inadequate follow-up, inadequate report of outcomes and use of alternative fixation types. In total, 28 studies were selected for inclusion (Table 1): 6 arthroscopic Bankart, 12 open Bankart and 11 open Latarjet with a total of
1652 patients. The majority of studies were case series with level IV evidence. There was a single level III study. A total of 1652 patients were analyzed with a mean age of 26.8 years and an overall mean follow-up of 160 months.

Least squares mean values and $95 \%$ confidence intervals for each outcome are listed in Table 2.

Six studies ${ }^{5,22-26}$ reported the results of 336 arthroscopic Bankart repairs. The mean Coleman score was 71.7 . The average age of included patients was 29.4 years (16-62 years) with a mean follow-up of 156 months (60-206 months). The dislocation rate was $15.1 \%$ with $20.2 \%$ of patients reporting subjective instability post-operatively. Four studies reported 


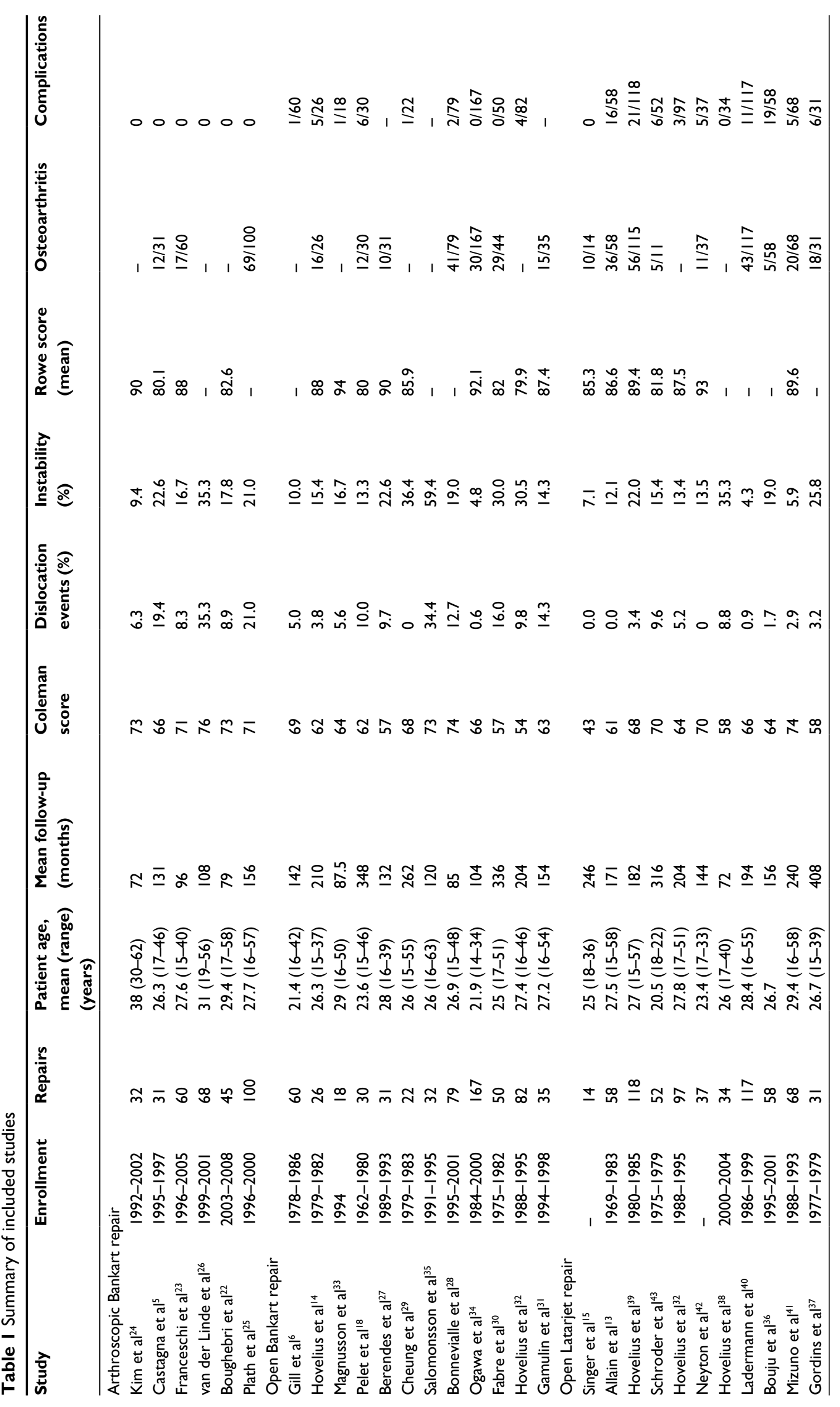


Table 2 Outcome analysis: least squares mean values with $95 \%$ confidence intervals

\begin{tabular}{|c|c|c|c|c|c|c|}
\hline Comparison & Dislocation, \% & Instability, \% & Rowe score & $\begin{array}{l}\text { Arthritis } \\
\text { (overall), \% }\end{array}$ & $\begin{array}{l}\text { Arthritis } \\
\text { (moderate to } \\
\text { severe), \% }\end{array}$ & Complication, \% \\
\hline \multirow[t]{2}{*}{ Arthroscopic Bankart (6) } & I5.I (9.9-20.3) & $20.2(11.7-28.7)$ & $85.5(80.1-90.8)$ & $45.9(24.4-67.4)$ & $21.0(8.4-33.6)$ & $0.0(-4.6 \text { to } 4.6)^{\mathrm{b}}$ \\
\hline & & & $4 / 6$ studies & $3 / 6$ studies & & $6 / 6$ studies \\
\hline \multirow[t]{2}{*}{ Open Bankart (12) } & $7.7(4.2-11.1)$ & $20.8(14.6-27.1)$ & 87.I (83.9-90.3) & $44.1(29.8-58.4)$ & II .8 (3.7-20.0) & $4.3(0.0-8.6)$ \\
\hline & & & $9 / 12$ studies & $7 / 12$ studies & & $9 / 12$ studies \\
\hline \multirow[t]{2}{*}{ Open Latarjet (I I) } & $2.7(-0.3-5.8)^{\mathrm{a}}$ & | 4.8 (8.6-20.9) & 87.9 (84.7-9|.2) & $42.0(29.3-54.8)$ & | 3.4 (5.9-20.8) & $10.6(6.6-14.7)^{b}$ \\
\hline & & & $7 / 11$ studies & $9 / 11$ studies & & II/II studies \\
\hline
\end{tabular}

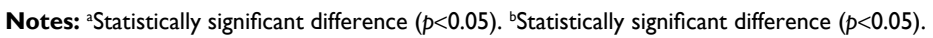

Rowe scores with an average of 85.5. Post-operative radiographic arthritis was documented in three studies for an overall rate of $45.9 \%$. No complications were reported in any of the studies.

Twelve studies ${ }^{6,14,18,27-35}$ investigated open Bankart repairs, for a total of 632 repairs. The mean Coleman score was 64.1. The average age of included patients was 24.8 years (14-55 years) with a mean follow-up of 182 months (60-492 months). The re-dislocation rate at 5 years was $7.7 \%$ with $20.8 \%$ of patients reporting subjective instability. Rowe scores were reported in nine studies with a mean score of 87.1. Radiographic arthritis was documented in seven studies for an overall rate of $44.1 \%$. A complication rate of $4.3 \%$ was recorded across nine studies.

Eleven studies ${ }^{13,15,32,36-43}$ reported the results of open Latarjet procedures for a total of 684 repairs. The mean Coleman score was 63.3. The average age of patients was 26.8 years (15-58 years) with a mean follow-up period of 212 months (60-420 months). The re-dislocation rate was $2.7 \%$ with $14.8 \%$ of patients complaining of subjective instability symptoms. Seven studies reported Rowe scores for an average score of 87.9. Radiographic arthritis was documented in $42.0 \%$ of patients in the nine studies that included this metric. Eleven studies reported complications, with an overall rate of $10.6 \%$.

There was a statistically significant reduction in re-dislocation comparing open Latarjet with arthroscopic Bankart $(p<0.001)$. However, there was no difference comparing arthroscopic Bankart to open Bankart or open Bankart to Latarjet procedures. There was no statistically significant difference between groups in rates of subjective instability or development of radiographic arthritis. Open Latarjet procedures had statistically significantly more complications compared to the arthroscopic Bankart $(p=0.002)$. Complications reported included superficial infection, superficial vein thrombosis, musculocutaneous neuropraxia, graft non-union, graft mal-union graft migration and intra-articular hardware.

\section{Discussion}

The primary goal of any stabilization procedure is to prevent recurrence of instability with the goal of improving function and to reduce long-term sequelae. In this review, the arthroscopic Bankart procedure had the highest absolute re-dislocation rate. Overall, there was a trend toward increasing stability with increasing invasiveness, although the only statistically significant result was between the arthroscopic Bankart and the open Latarjet. As the newest procedure of the three with the smallest patient cohort, the high re-dislocation rate may be partially related to a learning curve effect. The arthroscopic Bankart procedure has many technical variants that are known to influence outcomes, including beach chair versus lateral positioning, ${ }^{44}$ portal placement, ${ }^{45}$ fixation type $^{4}$ and placement and morphology of the labral repair. ${ }^{46}$ As such, it may be reasonable to expect that outcomes of the arthroscopic Bankart repair will improve as the optimal technique and indications are determined.

The open Bankart is conceptually a similar procedure to the arthroscopic Bankart with respect to the anatomic repair of the capsulolabral lesion. Interestingly, there was no significant difference noted comparing the arthroscopic Bankart to the open Bankart procedure. This could be interpreted in several ways. The first is that this supports the conclusion that the arthroscopic Bankart will continue to improve as techniques are developed to ensure proper repair characteristics. This is supported by Petrera et $\mathrm{al}^{47}$ who directly compared open vs arthroscopic Bankart repairs in studies utilizing similar repair configurations and found improved stability with arthroscopic surgery. However, there have also been several large meta-analyses comparing highquality trials, ${ }^{48,49}$ which conclude that there is a consistently higher risk of re-dislocation associated with the arthroscopic Bankart procedure. This suggests that there may in fact be an inherent difference between the arthroscopic and open Bankart procedures that cannot be improved with further technical nuances. 
The Latarjet repair mechanically restores stability in a completely different manner. In addition to providing stability by repairing the Bankart lesion, the coracoid transfer provides the most robust anterior restraint by managing bone loss of the glenoid (a known risk factor for re-dislocation ${ }^{11}$ ) and providing a dynamic soft-tissue restrain. This is reflected in the low re-dislocation rate and trend toward decreased subjective instability compared with either open or arthroscopic Bankart. However, despite increased surgical dissection and significant anatomical distortion, no statistically significant benefit was shown compared to the open Bankart repair. Unfortunately, given the limitations of a systematic review, we are unable to elucidate the contribution by patients with significant glenoid or humeral bone loss, ${ }^{50}$ which may be considered a contraindication to a standard Bankart repair. This highlights the importance of screening patients for bone deficiency and appropriate procedure selection when considering a soft-tissue repair. ${ }^{10}$

The high rate of persistent subjective instability, irrespective of procedure choice, at long-term follow-up is concerning. Despite the significant effect on patient function associated with recurrent subjective instability, it is difficult to interpret this result in the context of a broad range of patient age and functional expectation that are included in the analysis. Furthermore, the long-term functional and clinical significance of this finding is unknown especially in the setting of -high mean post-operative Rowe scores.

Dislocation arthropathy was initially introduced by Samilson and Prieto ${ }^{21}$ in 1938 after a high proportion of patients with soft-tissue tensioning procedures returned with painful, stiff shoulders at 13 years follow-up. However, arthropathy is also part of the natural history of anterior shoulder dislocation. Hovelius et al ${ }^{51}$ documented mild-to-moderate arthropathy in $20 \%$ of patients who sustained anterior dislocations at 10 years of follow-up. In a follow-up study, Hovelius and Saeboe ${ }^{52}$ documented the development of radiographic osteoarthritis in $56 \%$ of dislocators at 25 years follow-up. Importantly, they demonstrated that operative treatment reduced the overall rate of arthropathy, although patients with severe arthropathy were not salvaged by a stabilization procedure. In our study, there was a similarly high rate of radiographic arthropathy with all three reconstructive procedures. The relatively consistent rate of radiographic arthritic changes between groups supports the conclusion that, in the absence of obvious complication, dislocation arthropathy likely arises from the injury rather than the surgical procedure.

The relevance of dislocation arthropathy is clouded by poor correlation between radiographic and clinical arthritis. ${ }^{51}$
While multiple authors have documented high rates of radiographic evidence, the preponderance of cases in this analysis is in the mild-moderate spectrum. In a large, multi-centered trial of shoulder arthroplasty, Matsoukis et a ${ }^{53}$ documented that only $4 \%$ of 1542 arthroplasties were completed for dislocation arthropathy. Given the common prevalence of shoulder dislocation, it is clear that few will actually progress to debilitating symptomatic arthritis. However, Fabre et $a^{30}$ have shown that while some patients with severe glenohumeral arthritis have excellent functional scores, there is a trend toward worse outcomes with progression in the degree of radiographic arthritis.

The arthroscopic Bankart repairs had statistically less complications compared to the open Latarjet. While this is consistent with the literature regarding arthroscopic versus open surgery, ${ }^{54}$ the fact that there were no complications reported in any of the six studies likely indicates a reporting bias. Complications resulting from the Latarjet procedure are specific to the procedure itself (i.e., intra-articular hardware, graft non-union or mal-union) but can result in catastrophic failure with significant patient morbidity. Care must be taken with the interpretation of this statistic as the definitions used in this study exclude recurrent dislocation as a complication. In patient care, this represents a major source of patient morbidity and a failure of the procedure.

The possibility for revision surgery is an important consideration in any anterior stabilization procedure. While there is a higher rate of re-dislocation associated with both the open and arthroscopic Bankart procedures, these repairs are anatomy-preserving procedures. The arthroscopic Bankart procedure has had success as a revision procedure for both failed open and arthroscopic Bankart repairs, without outcomes similar to primary Bankart repairs. ${ }^{55,56}$ In comparison, the Latarjet procedure is a reconstructive rather than reparative procedure and is anatomy distorting. Revision surgery, while less common, is difficult and fraught with complications. ${ }^{57}$

Unfortunately, several valid limitations exist within the included studies. The first of these is the uniformly low level of evidence of included studies, a factor that has been shown to influence the results of systematic reviews and meta-analyses in this area, particularly with functional scores. ${ }^{48}$ The reported outcomes throughout the studies also vary considerably. Of the 28 included studies, only 21 reported Rowe scores, 19 reported radiographic arthrosis and 25 reported complications. Furthermore, although both the Rowe score and the Samilson-Prieto classification are commonly used in the shoulder literature, they are based on 
historical expert opinion and are known to have low interobserver reliability. ${ }^{58,59}$

Many of the remaining limitations of this study are consistent with those of systematic reviews in general. The patient population includes a broad selection of patients with different ages, functional demands, frequency of instability episodes and time to surgery, making the results difficult to apply to any particular patient. We are also unable to elucidate the mechanism of the recurrent dislocations and are therefore unable to differentiate between atraumatic events that may be secondary to surgical technique and traumatic instability events during participation in high-risk sports. Finally, the data extracted are unable to provide information on the prevalence of patient-specific risk factors or specific post-operative rehabilitation protocols.

\section{Conclusion}

Each of the three anterior shoulder stabilization repairs dramatically reduced the incidence of dislocation. However, $15-20 \%$ of patients will have subjective instability post-operatively. The Latarjet procedure had the lowest re-dislocation rate, which was significantly lower than that of the arthroscopic Bankart repair. Regardless of surgical procedure, nearly half of patients having surgery for anterior shoulder instability will develop radiographic glenohumeral arthritis.

Further investigation will be required in this area as the surgical technique for the arthroscopic Bankart continues to evolve and the Latarjet procedure continues its expansion from expert users in Europe to North America. Future studies are also needed to investigate the functional impact and clinical significance of dislocation arthropathy.

\section{Acknowledgment}

An author, their immediate family or any research foundation with which they are affiliated has received royalties, research support or fellowship support from Arthrex, Lippincott Williams and Wilkins, Smith \& Nephew and Conmed Linvatec.

\section{Disclosure}

The authors report no conflicts of interest in this work.

\section{References}

1. Zacchilli MA, Owens BD. Epidemiology of shoulder dislocations presenting to emergency departments in the United States. J Bone Joint Surg Am. 2010;92(3):542-549.

2. Bankart ASB. Recurrent dislocation of the shoulder-joint. $\mathrm{Br}$ Med J. 1925;2(3386):978.

3. Rowe CR. Acute and recurrent anterior dislocations of the shoulder. Orthop Clin North Am. 1980;11(2):253-270.
4. Kandziora F, Jager A, Bischof F, Herresthal J, Starker M, Mittlmeier T. Arthroscopic labrum refixation for post-traumatic anterior shoulder instability: suture anchor versus transglenoid fixation technique. Arthroscopy. 2000;16(4):359-366.

5. Castagna A, Markopoulos N, Conti M, Delle Rose G, Papadakou E, Garofalo R. Arthroscopic Bankart suture-anchor repair: radiological and clinical outcome at minimum 10 years of follow-up. Am J Sports Med. 2010;38(10):2012-2016.

6. Gill TJ, Micheli LJ, Gebhard F, Binder C. Bankart repair for anterior instability of the shoulder. Long-term outcome. J Bone Joint Surg Am. 1997;79(6):850-857.

7. Porcellini G, Campi F, Pegreffi F, Castagna A, Paladini P. Predisposing factors for recurrent shoulder dislocation after arthroscopic treatment. J Bone Joint Surg Am. 2009;91(11):2537-2542.

8. Shibata H, Gotoh M, Mitsui Y, et al. Risk factors for shoulder re-dislocation after arthroscopic Bankart repair. J Orthop Surg Res. 2014;9:53.

9. Latarjet $M$. Technigues chirurgicales dans le traitement de la luxation r'ecidivante de l'epaule (Ant'ero-interne) [Surgical technics in the treatment of recurrent dislocation of the shoulder (antero-internal)]. Lyon Chir. 1965;61:313-318. French.

10. Burkhart SS, De Beer JF. Traumatic glenohumeral bone defects and their relationship to failure of arthroscopic Bankart repairs: significance of the inverted-pear glenoid and the humeral engaging Hill-Sachs lesion. Arthroscopy. 2000;16(7):677-694.

11. Lo IK, Bishop JY, Miniaci A, Flatow EL. Multidirectional instability: surgical decision making. Instr Course Lect. 2004;53:565-572.

12. Wellmann M, De Ferrari H, Smith T, et al. Biomechanical investigation of the stabilization principle of the Latarjet procedure. Arch Orthop Trauma Surg. 2012;132(3):377-386.

13. Allain J, Goutallier D, Glorion C. Long-term results of the Latarjet procedure for the treatment of anterior instability of the shoulder. J Bone Joint Surg Am. 1998;80(6):841-852.

14. Hovelius LK, Sandstrom BC, Rosmark DL, Saebo M, Sundgren KH, Malmqvist BG. Long-term results with the Bankart and Bristow-Latarjet procedures: recurrent shoulder instability and arthropathy. J Shoulder Elbow Surg. 2001;10(5):445-452.

15. Singer GC, Kirkland PM, Emery RJ. Coracoid transposition for recurrent anterior instability of the shoulder. A 20-year follow-up study. J Bone Joint Surg Br. 1995;77(1):73-76.

16. Kiss J, Mersich I, Perlaky GY, Szollas L. The results of the Putti-Platt operation with particular reference to arthritis, pain, and limitation of external rotation. J Shoulder Elbow Surg. 1998;7(5):495-500.

17. van der Zwaag HM, Brand R, Obermann WR, Rozing PM. Glenohumeral osteoarthrosis after Putti-Platt repair. J Shoulder Elbow Surg. 1999;8(3):252-258.

18. Pelet S, Jolles BM, Farron A. Bankart repair for recurrent anterior glenohumeral instability: results at twenty-nine years' follow-up. J Shoulder Elbow Surg. 2006;15(2):203-207.

19. Coleman BD, Khan KM, Maffulli N, Cook JL, Wark JD. Studies of surgical outcome after patellar tendinopathy: clinical significance of methodological deficiencies and guidelines for future studies. Victorian institute of sport tendon study group. Scand J Med Sci Sports. 2000;10(1): 2-11.

20. Rowe CR, Patel D, Southmayd WW. The Bankart procedure: a long-term end-result study. J Bone Joint Surg Am. 1978;60(1):1-16.

21. Samilson RL, Prieto V. Dislocation arthropathy of the shoulder. J Bone Joint Surg Am. 1983;65(4):456-460.

22. Boughebri O, Maqdes A, Moraiti C, Dib C, Leclere FM, Valenti P. Results of 45 arthroscopic Bankart procedures: does the ISIS remain a reliable prognostic assessment after 5 years? Eur J Orthop Surg Traumatol. 2015;25(4):709-716.

23. Franceschi F, Papalia R, Del Buono A, Vasta S, Maffulli N, Denaro V. Glenohumeral osteoarthritis after arthroscopic Bankart repair for anterior instability. Am J Sports Med. 2011;39(8):1653-1659.

24. Kim SJ, Kim TW, Moon HK, Chang WH. A combined transglenoid and suture anchor technique for bony Bankart lesions. Knee Surg Sports Traumatol Arthrosc. 2009;17(12):1443-1446. 
25. Plath JE, Aboalata M, Seppel G, et al. Prevalence of and risk factors for dislocation narthropathy: radiological long-term outcome of arthroscopic Bankart repair in 100 shoulders at an average 13 year follow-up. Am J Sports Med. 2015;43(5):1084-1090.

26. van der Linde JA, van Kampen DA, Terwee CB, Dijksman LM, Kleinjan G, Willems WJ. Long-term results after arthroscopic shoulder stabilization using suture anchors: an 8- to 10-year follow-up. Am J Sports Med. 2011;39(11):2396-2403.

27. Berendes TD, Wolterbeek R, Pilot P, Verburg H, te Slaa RL. The open modified Bankart procedure: outcome at follow-up of 10 to 15 years. J Bone Joint Surg Br. 2007;89(8):1064-1068.

28. Bonnevialle N, Mansat P, Bellumore Y, Mansat M, Bonnevialle P. Selective capsular repair for the treatment of anterior-inferior shoulder instability: review of seventy-nine shoulders with seven years' average follow-up. J Shoulder Elbow Surg. 2009;18(2):251-259.

29. Cheung EV, Sperling JW, Hattrup SJ, Cofield RH. Long-term outcome of anterior stabilization of the shoulder. J Shoulder Elbow Surg. 2008; 17(2):265-270.

30. Fabre T, Abi-Chahla ML, Billaud A, Geneste M, Durandeau A. Longterm results with Bankart procedure: a 26-year follow-up study of 50 cases. J Shoulder Elbow Surg. 2010;19(2):318-323.

31. Gamulin A, Dayer R, Lubbeke A, Miozzari H, Hoffmeyer P. Primary open anterior shoulder stabilization: a long-term, retrospective cohort study on the impact of subscapularis muscle alterations on recurrence. BMC Musculoskelet Disord. 2014;15:45.

32. Hovelius L, Vikerfors O, Olofsson A, Svensson O, Rahme H. BristowLatarjet and Bankart: a comparative study of shoulder stabilization in 185 shoulders during a seventeen-year follow-up. J Shoulder Elbow Surg. 2011;20(7):1095-1101.

33. Magnusson L, Ejerhed L, Rostgard-Christensen L, et al. A prospective, randomized, clinical and radiographic study after arthroscopic Bankart reconstruction using 2 different types of absorbable tacks. Arthroscopy. 2006;22(2):143-151.

34. Ogawa K, Yoshida A, Matsumoto H, Takeda T. Outcome of the open Bankart procedure for shoulder instability and development of osteoarthritis: a 5- to 20-year follow-up study. Am J Sports Med. 2010;38(8): $1549-1557$.

35. Salomonsson B, Abbaszadegan H, Revay S, Lillkrona U. The Bankart repair versus the Putti-Platt procedure: a randomized study with WOSI score at 10-year follow-up in 62 patients. Acta Orthop. 2009;80(3):351-356.

36. Bouju Y, Gadea F, Stanovici J, Moubarak H, Favard L. Shoulder stabilization by modified Latarjet-Patte procedure: results at a minimum 10 years' follow-up, and role in the prevention of osteoarthritis. Orthop Traumatol Surg Res. 2014;100(4 Suppl):S213-S218.

37. Gordins V, Hovelius L, Sandstrom B, Rahme H, Bergstrom U. Risk of arthropathy after the Bristow-Latarjet repair: a radiologic and clinical thirty-three to thirty-five years of follow-up of thirty-one shoulders. J Shoulder Elbow Surg. 2015;24(5):691-699.

38. Hovelius L, Sandstrom B, Olofsson A, Svensson O, Rahme H. The effect of capsular repair, bone block healing, and position on the results of the Bristow-Latarjet procedure (study III): long-term follow-up in 319 shoulders. J Shoulder Elbow Surg. 2012;21(5):647-660.

39. Hovelius L, Sandstrom B, Saebo M. One hundred eighteen BristowLatarjet repairs for recurrent anterior dislocation of the shoulder prospectively followed for fifteen years: study II-the evolution of dislocation arthropathy. J Shoulder Elbow Surg. 2006;15(3):279-289.

40. Ladermann A, Lubbeke A, Stern R, Cunningham G, Bellotti V, Gazielly DF. Risk factors for dislocation arthropathy after Latarjet procedure: a long-term study. Int Orthop. 2013;37(6):1093-1098.

41. Mizuno N, Denard PJ, Raiss P, Melis B, Walch G. Long-term results of the Latarjet procedure for anterior instability of the shoulder. J Shoulder Elbow Surg. 2014;23(11):1691-1699.
42. Neyton L, Young A, Dawidziak B, et al. Surgical treatment of anterior instability in rugby union players: clinical and radiographic results of the Latarjet-Patte procedure with minimum 5-year follow-up. J Shoulder Elbow Surg. 2012;21(12):1721-1727.

43. Schroder DT, Provencher MT, Mologne TS, Muldoon MP, Cox JS. The modified Bristow procedure for anterior shoulder instability: 26-year outcomes in Naval Academy Midshipmen. Am J Sports Med. 2006;34(5):778-786.

44. Frank RM, Saccomanno MF, McDonald LS, Moric M, Romeo AA, Provencher MT. Outcomes of arthroscopic anterior shoulder instability in the beach chair versus lateral decubitus position: a systematic review and meta-regression analysis. Arthroscopy. 2014;30(10):1349-1365.

45. Dwyer T, Petrera M, White LM, et al. Trans-subscapularis portal versus low-anterior portal for low anchor placement on the inferior glenoid fossa: a cadaveric shoulder study with computed tomographic analysis. Arthroscopy. 2015;31(2):209-214.

46. Kim JY, Chung SW, Kwak JY. Morphological characteristics of the repaired labrum according to glenoid location and its clinical relevance after arthroscopic Bankart repair: postoperative evaluation with computed tomography arthrography. Am J Sports Med. 2014; 42(6):1304-1314.

47. Petrera M, Patella V, Patella S, Theodoropoulos J. A meta-analysis of open versus arthroscopic Bankart repair using suture anchors. Knee Surg Sports Traumatol Arthrosc. 2010;18(12):1742-1747.

48. Lenters TR, Franta AK, Wolf FM, Leopold SS, Matsen FA 3rd. Arthroscopic compared with open repairs for recurrent anterior shoulder instability. A systematic review and meta-analysis of the literature. J Bone Joint Surg Am. 2007;89(2):244-254.

49. Mohtadi NG, Bitar IJ, Sasyniuk TM, Hollinshead RM, Harper WP. Arthroscopic versus open repair for traumatic anterior shoulder instability: a meta-analysis. Arthroscopy. 2005;21(6):652-658.

50. Lo IK, Parten PM, Burkhart SS. The inverted pear glenoid: an indicator of significant glenoid bone loss. Arthroscopy. 2004;20(2):169-174.

51. Hovelius L, Augustini BG, Fredin H, Johansson O, Norlin R, Thorling J. Primary anterior dislocation of the shoulder in young patients. A ten-year prospective study. J Bone Joint Surg Am. 1996;78(11):1677-1684.

52. Hovelius L, Saeboe M. Neer award 2008: arthropathy after primary anterior shoulder dislocation - 223 shoulders prospectively followed up for twenty-five years. J Shoulder Elbow Surg. 2009;18(3):339-347.

53. Matsoukis J, Tabib W, Guiffault P, Walch G. Prothese d'epaule sur omarthrose secondaire a une instabilite anterieure non operee: A propos d'une serie de 28 cas [Shoulder arthroplasty for osteoarthritis after prior surgery for anterior instability: a report of 27 cases]. Revue de Chirurgie Orthopedique et Reparatrice de l Appareil Moteur. 2003;89(7):580-592. French.

54. Owens BD, Harrast JJ, Hurwitz SR, Thompson TL, Wolf JM. Surgical trends in Bankart repair: an analysis of data from the American board of orthopaedic surgery certification examination. Am J Sports Med. 2011;39(9):1865-1869.

55. Abouali JA, Hatzantoni K, Holtby R, Veillette C, Theodoropoulos J. Revision arthroscopic Bankart repair. Arthroscopy. 2013;29(9):1572-1578.

56. Millar NL, Murrell GA. The effectiveness of arthroscopic stabilisation for failed open shoulder instability surgery. J Bone Joint Surg Br. 2008;90(6):745-750.

57. Young DC, Rockwood CA Jr. Complications of a failed Bristow procedure and their management. J Bone Joint Surg Am. 1991;73(7):969-981.

58. Ilg A, Bankes MJ, Emery RJ. The intra- and inter-observer reliability of the Samilson and Prieto grading system of glenohumeral arthropathy. Knee Surg Sports Traumatol Arthrosc. 2001;9(3):187-190.

59. Skare O, Schroder CP, Mowinckel P, Reikeras O, Brox JI. Reliability, agreement and validity of the 1988 version of the Rowe Score. J Shoulder Elbow Surg. 2011;20(7):1041-1049. 
The Open Access Journal of Sports Medicine is an international, peer-reviewed, open access journal publishing original research, reports, reviews and commentaries on all areas of sports medicine. The journal is included on PubMed. The manuscript management system is completely online and includes a very quick and fair peer-review system. Visit http://www.dovepress.com/testimonials.php to read real quotes from published authors.

Submit your manuscript here: http://www.dovepress.com/open-access-journal-of-sports-medicine-journal 